\title{
Pancreatitis During Pregnancy Revealing Solid Pseudopapillary Tumor of the Pancreas: Case Report
}

\section{Imen Ganzoui ${ }^{1 *}$, Dorsaf Nouri ${ }^{1}$, Mariem Balti ${ }^{1}$ and Karim Ayed ${ }^{2 *}$}

${ }^{1}$ Radiology Department, University of Tunis El Manar, Habib Bougatfa Hospital

Bizerta, Tunisia

${ }^{2}$ Surgery Department, University of Tunis El Manar, Habib Bougatfa Hospital

Bizerta, Tunisia

*Corresponding Author: Imen Ganzoui, Head of Radiology Department, University

of Tunis El Manar, Habib Bougatfa Hospital Bizerta, Tunisia.
Received: September 13, 2021

Published: September 23, 2021

(C) All rights are reserved by Imen Ganzoui., et al.

\section{Abstract}

A 26-year-old primigravid patient, at 11 weeks of gestation, was referred to the digestive surgery department for acute pancreatitis, the abdominal ultrasound revealed a solid pancreatic mass. The MRI showed a well circumscribed tumor in the body of the pancreas with no local or distant invasion. The diagnosis of solid pseudopapillary tumor of the pancreas (SPTP) was then suspected. After multi-disciplinary reunion including the patient and her husband, the decision was to operate the patient but first medically interrupt the pregnancy. The operation consisted in left pancreatectomy with conservation of the spleen gland. The histopathological examination confirmed the diagnosis of SPTP. There was no complication or recurrence after 10 months of follow up.

Keywords: Frantz Tumor; Pregnant Woman; Pancreas; Solid Pseudopapillary Tumor; Pancreatitis

\section{Abbreviation}

SPTP: Solid Pseudopapillary Tumor of the Pancreas.

\section{Introduction}

Solid pseudopapillary tumor of the pancreas (SPTP) is rare, counting less than $2 \%$ of pancreatic tumors, and mostly occurring in young woman, especially in their $2^{\text {nd }}$ decade [1].

SPTP during pregnancy is exceptional and presents a threat both to the mother and the fetus due to the potential for accelerated growth during this time [2].

A multidisciplinary approach with respect to the pregnant patient's diagnosis, indications, and timing of surgery is necessary in ensuring the best possible outcomes for both the mother and the child.

\section{Case Presentation}

A 26-year-old woman, primigravid 11 weeks of gestation, admitted for acute epigastric pain with vomiting.

Physical examination was normal.

The obstetrical check-up and the fetal ultrasound were also normal.

Blood test showed leukocytosis, cytolysis and cholestasis. Amylase and lipase tests were high thus revealing an acute pancreatitis.

Abdominal ultrasound was performed looking for biliary lithiasis demonstrated a well circumscribed non homogenous tumor located to the body of the pancreas. There was no lithiasis in the gallbladder. 
Due to radiation consideration during pregnancy, an MRI was then indicated for further characterization of the mass. It revealed a solid lesion with no cystic or hemorrhagic component, located in the body of the pancreas, high intense signal on T2/T2FS weighted images, hyper intense signal on diffusion, heterogeneously enhancing after injection of contrast material progressively and mostly on the late phase, measuring about $5.5 \times 4.5 \mathrm{~cm}$ (Figure 1 ).
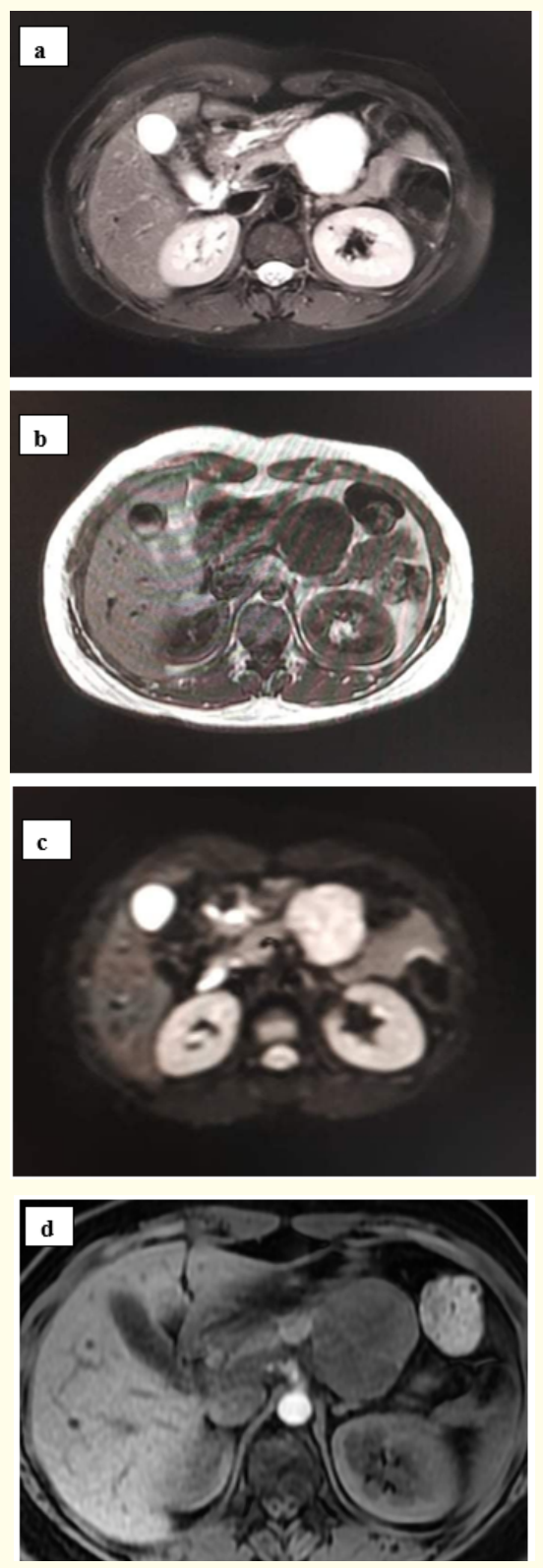
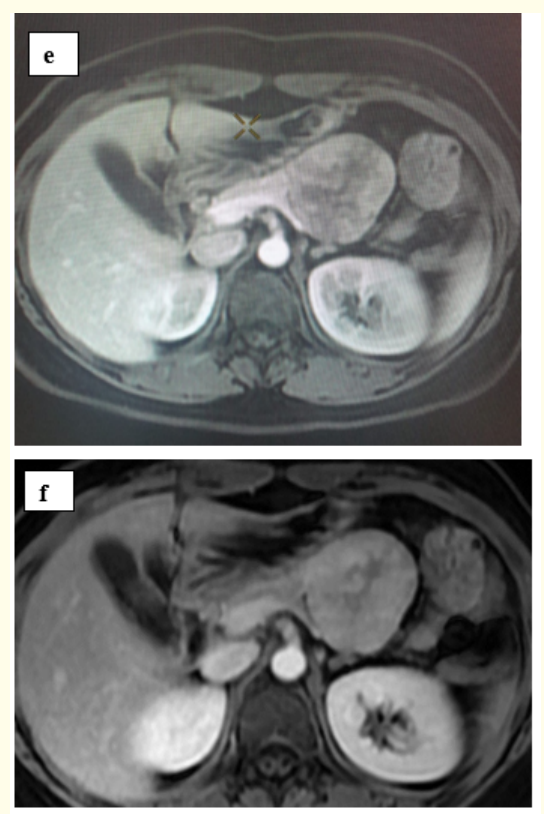

Figure 1: T2FS (a), T1 (b), Diffusion B1000 (c), contrast-enhanced T1-weighted images; (d): non contrast (d), arterial (e) late phase (f), well circumscribed mass in the body of the pancreas progressively enhancing with rare focal areas of necrosis.

There was no sign of metastatic location on the rest of the abdomen.

Tumor markers including carcinoembryonic antigen $(\mathrm{CEA}=1$ $\mathrm{ng} / \mathrm{ml}$ ), cancer antigen 125 (CA125 = $25 \mathrm{U} / \mathrm{ml}$ ), and carbohydrate antigen $199(\mathrm{CA} 199=3 \mathrm{U} / \mathrm{ml})$ were within normal ranges.

Theses imaging features were suggestive for either SPTP (mostly solid) or non-functional endocrine tumor of the pancreas.

After multi-disciplinary reunion with the patient and her husband consent, the pregnancy had to be medically interrupted before surgical intervention consisting of left pancreatectomy with conservation of the splenic vessels and the spleen (Figure 2).

Histological examination confirmed the diagnosis of solid pseudopapillary tumor of the pancreas.

There were no postoperative complications. There was no recurrence after a follow up of 10 months. 


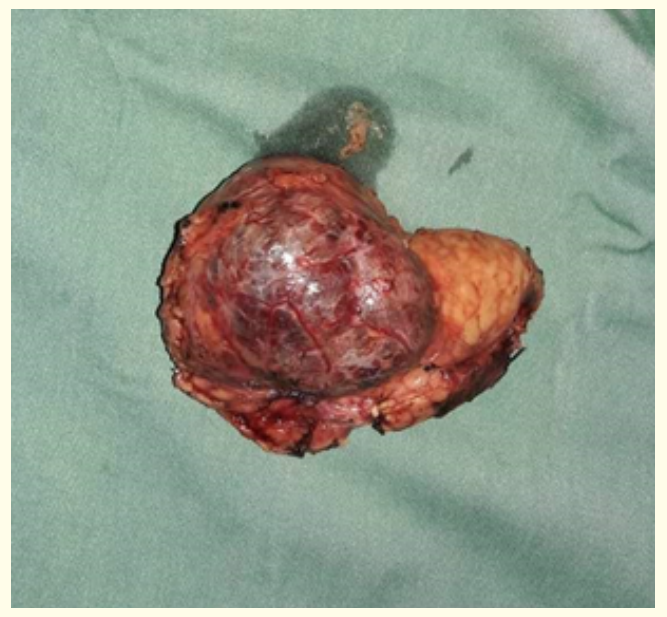

Figure 2: Left pancreatectomy "en bloc" with the tumor.

\section{Discussion}

- $\quad$ SPTP was first described by Frantz in 1959 and is rare in pregnancy. To the best of our knowledge, there are only twelve cases of SPTP found and treated during pregnancy [3].

- $\quad$ SPTP mostly occurs in young woman, aged between 20 and 40 years old, counting for $95 \%[1,2]$.

- This tumor is located in the body and the tail of the pancreas in about $64 \%$ of cases [1].

- Symptoms are subtle and most of time non-specific. Our patient, like many of the other examples, presented with relatively vague symptoms of abdominal discomfort, nausea and vomiting. These may be falsely associated with pregnancy in patients presenting for routine prenatal care. In the absence of a focused physical examination and appropriate diagnostic workup, SPTP during pregnancy may go undetected for some time. It may be also revealed incidentally when performing imaging exam for another purpose.

- We found no previous case of pancreatitis associated with SPTP in pregnant woman in the literature.

- Imaging techniques (CT/MRI) show typically necrosis, hemorrhage, solid and cystic components $[4,7]$.
- Diagnosis is often confirmed by histological examination using histochemistry.

- The prognosis is good, especially after complete resection, with low risk of malignancy. However, it requires a long term follow up to detect any recurrence.

- The prognosis is good, with low risk of malignancy. The malignant potential of SPTP is reported to be $10 \%-15 \%$ [8].

- Biopsy is not recommended if the diagnosis of SPTP is suspected due to the risk of dissemination [2].

- Histological examination after a complete resection remain the gold standard for diagnosis.

- $\quad$ Microscopically, tumors cells are arranged around fibrovascular stem making a pseudopapillary pattern. Focal areas of necrosis and hemorrhage are often present, and mitosis are rare.

- Positivity for progesterone receptors is sometimes reported, which may explain female predominance [6].

- $\quad$ Surgery remains the cornerstone of the treatment. The technique depends on the size and the location of the tumor. Invasion of adjacent organs plays also a crucial role in determining the optimal procedure. For tumors limited to the pancreas, complete surgical resection is curative in more than $95 \%$ of cases. If there is any metastatic lesion or any recurrence, surgery is still the treatment to consider $[1,4]$.

- A long-term follow-up is required to detect any recurrence which occurs in about 10 to $15 \%$ and which needs surgical intervention $[1,6,7]$.

- There is no treatment guideline for pregnant women with SPTP, and therefore, decisions on the optimal timing for surgical management can be challenging. Surgical intervention during the first trimester may be associated with spontaneous abortion or poor fetal outcome, including congenital anomalies [5].

- The second trimester is the preferred time window for surgical intervention for resecable SPTP [9].

- $\quad$ Several cases of ruptures of SPTP have been reported causing maternal instability during pregnancy. In this situation, emergency surgical intervention is performed, regardless of the gestational age [9]. 


\section{Conclusion}

Management of SPTP is more difficult during pregnancy because a balance between maternal and fetal well-being and surgical intervention for the tumor must be considered.

Clinical findings, radiologic screening results, general surgery consultation, and tumor complications, along with maternal and fetal well-being, should all be assessed to determine the optimal timing of surgery. There is also limited clinical experience described in the literature due to the rarity of pancreatic tumors in pregnancy.

\section{Conflict of Interest}

The authors declare that there is no conflict of interest.

\section{Bibliography}

1. Jakhlal N., et al. "Tumeur pseudopapillaire et solide du pancréas : à propos d'un cas et revue de la literature [Solid pseudopapillary tumour of the pancreas: about a case and review of the literature]". Pan African Medical Journal 24 (2016): 104.

2. D T Alexandrescu., et al. "Metastatic solid-pseudopapillary tumour of the pancreas: Clinico-biolgical correlates and management". Clinical Oncology 17.5 (2005): 358-363.

3. SC Huang., et al. "Spontaneous rupture of solidpseudopapillary neoplasm of the pancreas during pregnancy". Obstetrics and Gynecology 121 (2013): 486-488.

4. C Levy., et al. "Solid pseudopapillary pancreatic tumor in pregnancy. A case report". Journal of Reproductive Medicine 49.1 (2004): 61-64.

5. F MacDonald., et al. "Surgical therapy of a large pancreatic solid-pseudopapillary neoplasm during pregnancy". BMJ Case Reports (2014).

6. TT Huang., et al. "Solid Pseudopapillary Neoplasm of Pancreas in Pregnancy Treated with Tumor Enucleation: Case Report and Review of the Literature". Hindawi Case Reports in Obstetrics and Gynecology (2018): 3.

7. Yee AM., et al. "Solid pseudopapillary neoplasm of the pancreas head in a pregnant woman: Safe pancreaticoduodenectomy postpartum". Journal of Surgical Case Reports 2015 (2015): : pii: rjv108.

8. Chhabra M and Daver RG. "Solid pseudopapillary epithelial neoplasm of pancreas in pregnancy: Case report of a rare cooccurrence". JMSCR 8 (2017): 26978-26983.
9. "Surgical therapy of a large pancreatic solid-pseudopapillary neoplasm during pregnancy". BMJ Case Reports (2014):

Volume 3 Issue 10 October 2021

(C) All rights are reserved by Imen Ganzoui., et al. 